\title{
Electrical Stimulation Therapy in Chronic Functional Constipation: Five Years' Experience in Patients Refractory to Biofeedback Therapy and With Rectal Hyposensitivity
}

\begin{abstract}
Kee Wook Jung, Dong-Hoon Yang, In Ja Yoon, So Young Seo, Hyun Sook Koo, Hyo Jeong Lee, Ho Su Lee, Ji-Beom Kim, Jong Wook Kim, Soo Kyung Park, Sang Hyoung Park, Kyung Jo Kim, Byong Duk Ye, Jeong-Sik Byeon, Hwoon-Yong Jung, Suk-Kyun Yang, Jin-Ho Kim and Seung-Jae Myung*
\end{abstract}

Asan Digestive Disease Research Institute, Department of Gastroenterology, Asan Medical Center, University of Ulsan College of Medicine, Seoul, Korea

\section{Background/Aims}

Biofeedback therapy (BFT) can be unsuccessful in constipated patients, even those with pelvic floor dysfunction. Electrical stim ulation therapy (EST) has been introduced as a novel therapeutic modality in patients with chronic constipation, especially those who have rectal hyposensitivity. We evaluated the efficacy of EST based on five years' clinical experience.

\section{Methods}

From January 2002 to February 2007, 159 patients underwent EST. After exclusion of 12 drop-outs, 147 (M:F = 61:86, 49 \pm 17 years) finished all treatment sessions. Among them, 88 ( $M: F=29: 59,49 \pm 17$ years) were refractory to BFT without rectal hyposensitivity $(\mathrm{RH})$, and $59(\mathrm{M}: \mathrm{F}=32: 27,54 \pm 17$ years) were those with $\mathrm{RH}$.

\section{Results}

The overall response to EST was $59.2 \%$ (87/147) by per-protocol analysis. In the EST-responsive group, overall satisfaction improved significantly (from $7.3 \pm 3.0$ to $4.3 \pm 2.5, P<0.05$ ). Subgroup analysis showed that the response rate was $64.8 \%$ $(57 / 88)$ in patients refractory to BFT without $\mathrm{RH}$, and $50.8 \%(30 / 59)$ in those with $\mathrm{RH}$.

\section{Conclusions}

EST may have additional therapeutic efficacy in patients who are refractory to BFT. EST may also be effective in patients with $\mathrm{RH}$, including restoration of rectal sensation. Therefore, EST could be considered as an alternative choice in patients refractory to BFT and with or without RH.

(J Neurogastroenterol Motil 2013;19:366-373)

\section{Key Words}

Biofeedback; Constipation; Electric stimulation therapy

Received: January 28, 2013 Revised: March 12, 2013 Accepted: March 19, 2013

(C) This is an Open Access article distributed under the terms of the Creative Commons Attribution Non-Commercial License (http://creativecommons org/licenses/by-nc/3.0) which permits unrestricted non-commercial use, distribution, and reproduction in any medium, provided the original work is properly cited.

*Correspondence: Seung-Jae Myung, MD

Department of Internal Medicine, Asan Medical Center, University of Ulsan College of Medicine, 88, Olympic-ro 43-gil, Songpa-gu Seoul 138-736, Korea

Tel: +82-2-3010-3917, Fax: +82-2-476-0824, E-mail: sjmyung@amc.seoul.kr

Financial support: This study was supported by a grant of the Korean Health Technology R\&D Project, Ministry of Health \& Welfare, Republic of Korea (No. HI10C2014), the Bio \& Medical Technology Development Program of the National Research Foundation (NRF) funded by the Korean government (MEST) (2011-0019632), and the Asan Institute for Life Sciences (No. 2013-559).

Conflicts of interest: None (This study has not been published except for DDW 2008 as a poster)

Author contributions: Kee Wook Jung: writing of manuscript, analysis and collection of data. Seung-Jae Myung: writing of manuscript. Dong-Hoon Yang, In Ja Yoon, So Young Seo, Hyun Sook Koo, Hyo Jeong Lee, Ho Su Lee, Ji-Beom Kim, Jong Wook Kim, Soo Kyung Park, Sang Hyoung Park, Kyung Jo Kim, Byong Duk Ye, Jeong-Sik Byeon, Hwoon-Yong Jung, Suk-Kyun Yang and Jin-Ho Kim: writing of manuscript and collection of data. 


\section{Introduction}

Electrical stimulation therapy (EST) of the gastrointestinal tract has been used to treat gastric motor dysfunction since 1963. ${ }^{1,2}$ EST in the colon may be an alternative to conventional treatments, such as biofeedback, and surgery. ${ }^{3-5}$ Most EST procedures of the colon, such as sacral nerve stimulation and intramural stimulation, are regarded as invasive. ${ }^{3}$ EST using an anal plug is a non-invasive method having been used to treat urinary incontinence since the 1970s. ${ }^{6}$ As EST may rehabilitate the pelvic floor muscle, it has been tested in defecation disorders. EST using an anal plug has been shown successful in up to $60 \%$ of patients with fecal incontinence. ${ }^{7,8}$

EST using an anal plug has been found as effective as conventional biofeedback treatment in patients with rectal hyposensitivity (RH). ${ }^{4,5}$ This method has also been used to treat patients with functional constipation, regardless of RH, including patients refractory to conventional biofeedback therapy. ${ }^{9}$

We have therefore assessed the effect of EST in patients with chronic constipation and compared EST with BFT. We also performed multivariate analysis to determine factors prognostic of successful treatment with EST.

\section{Materials and Methods}

\section{Patients}

Patients with chronic functional defecatory disorder who visited Asan Medical Center, Seoul, Korea, between January 2002 and February 2007 were enrolled. Indications for EST included patients diagnosed with functional defecatory disorder who failed to respond to conventional biofeedback therapy. Most of these patients had first been treated with medication, and patients diagnosed by ROME II or who had pelvic floor dyssynergia (PFD) were indicated for BFT. PFD was diagnosed by anorectal manometry, balloon expulsion test, defecography, and colon transit time study. ROME II criteria for PFD included inappropriate contraction or failure to relax the pelvic floor muscles during repeated attempts to defecate. All enrolled patients satisfied ROME II criteria. ${ }^{10}$ Organic diseases were excluded by colonoscopy or barium enema. Patients with a history of diabetes mellitus, hypothyroidism, psychiatric disturbance, previous history of intestinal surgery, or drug abuse were excluded. Patients with slow transit time alone, another subtype of chronic constipation, were also ex- cluded, but patients with both slow transit time and PFD were included. Patients with a pacemaker or implantable cardioverter-defibrillator were excluded, as were patients with a history of current or recent anal bleeding.

Loss of follow-up was defined when patients who had undergone EST once did not undergo subsequent EST sessions regardless of patients' will. This retrospective analysis was approved by institutional review board of Asan Medical Center.

\section{Definition of Pelvic Floor Dyssynergia and Rectal Hyposensitivity}

Patients, who met the diagnostic criteria of ROME II, were diagnosed with PFD if they had dyssynergia on anorectal manometry and an inability to expel a $50 \mathrm{~mL}$ water filled balloon in 5 minutes. Rectal hyposensitivity ( $\mathrm{RH}$ ) was defined as the point when the patient could no longer feel any sensation of a desire to defecate in spite of the balloon being inflated up to $80 \mathrm{~mL}$ and when more than $90 \mathrm{~mL}$ of air inflation was needed to feel a sensation of a desire to defecate, which is currently the cut-off value at our institution.

\section{Anorectal Manometry}

Before enrollment, all patients underwent anorectal manometry to determine the cause of chronic constipation. All standard procedures were performed by one experienced nurse, and PFD was diagnosed by interpreting pressure curves during the bearing down phase. Manometry was performed using a stationary pull through technique with a microcapillary infusion system, Zinetic polyvinyl catheters (Medtronic, Denmark) and Polygramnet software (Medtronic, Denmark).

\section{Balloon Expulsion Test}

Following anorectal manometry, all patients were told to expel a $50 \mathrm{~mL}$ adult anorectal latex water-filled balloon. Five minutes of straining were allowed, after of which 'failure of balloon expulsion' was diagnosed.

\section{Colon Transit Time Study}

For the colon transit time (CTT) measurement, we used capsules of hardened gelatine filled with barium-impregnated polyethylene markers (pellets). Seven days before examination, any kind of medications and enemas that could influence the examination results were stopped. A Sitzmarker capsule containing 24 radiopaque, 1- to 2-mm thick rings (Sitzmarker, Lafayette Pharmaceutical, Inc, Forth Worth, TX, USA) was taken one per each 
day for 3 days. On the fourth day, simple erect film was checked. CTTS was measured following the previously published method. $^{11}$

Usually in normal CTT, at least $80 \%$ of the markers should be passed spontaneously by the fifth day, and all markers should be expelled by the seventh day. Pelvic retention of the markers is consistent with pelvic outlet obstruction, whereas diffuse scatter is more consistent with colonic inertia.

\section{Electrical Stimulation Therapy}

EST was performed using a computer with software, an electrical generator, a monitor, and an anal plug (Kontinence Clinical HMT 2000; HMT, Seoul, Korea), with all procedures performed by one well-trained, experienced nurse. Each EST session took 24 minutes, with 12 sessions per 2-week cycle. In each session, an anal plug was inserted into the patient's anus. Stimulatory parameters included a pulse width of 360-960 $\mu \mathrm{sec}$, a frequency of 2-110 Hz, and an amplitude of 30-35 V with the voltage adjusted to each patient's threshold of electrical pain sense. The clinical HMT 2000 could provide a variant mode, which was developed to overcome methodical limits and avoid problems such as discomfort and muscle fatigue. The main characteristics of variant mode were optimization of the stimulation waveform, synchronization to adjust energy, and automatic energy control by the structure of the double channel electrodes. ${ }^{12}$

\section{Indications of Electrical Stimulation Therapy}

We hypothesized the indications for EST as follows: patients who are refractory to biofeedback therapy, and patients who have

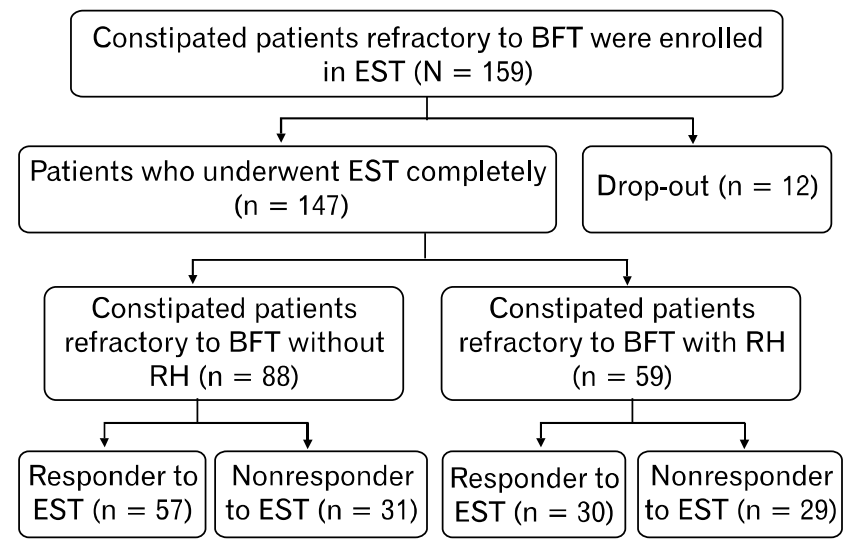

Figure 1. Study protocols of electrical stimulation therapy. BFT, biofeedback therapy; EST, electrical stimulation therapy; $\mathrm{RH}$, rectal hyposensitivity. rectal hyposensitivity.

\section{Study Design: Protocols}

Before EST, patients underwent CTT study, anorectal manometry (ARM) and balloon expulsion tests (BET). After EST, patients again underwent ARM and BET to define the differences in objective parameters after the therapy. Before and after EST, all enrolled patients completed questionnaires addressing subjective parameters during defecation, including overall satisfaction, straining, feelings of incomplete evacuation, and feelings of obstruction. Overall satisfaction was graded from 0 (very unsatisfactory) to 10 (very satisfactory); other subjective parameters were scored from 0 (absent) to 10 (very severe). Each patient's response to EST was recorded upon the last session of EST and scored as yes or no. Treatment outcome was assessed as responsive or non-responsive, based on the results of symptom questionnaires. EST was defined as achieving a good response when at least a two point improvement was seen in the global defecatory satisfactory score compared with the score prior to EST.

\section{Statistical Methods}

Assessment of treatment efficacy was analyzed at the end of each session. All statistical analyses were performed using SPSS version 13.0 (SPSS Inc., Illinois, USA). The Mann-Whitney U test and the Wilcoxon rank sum test were used to analyze continuous parameters, while the Chi-square test was used to analyze non-continuous parameters.

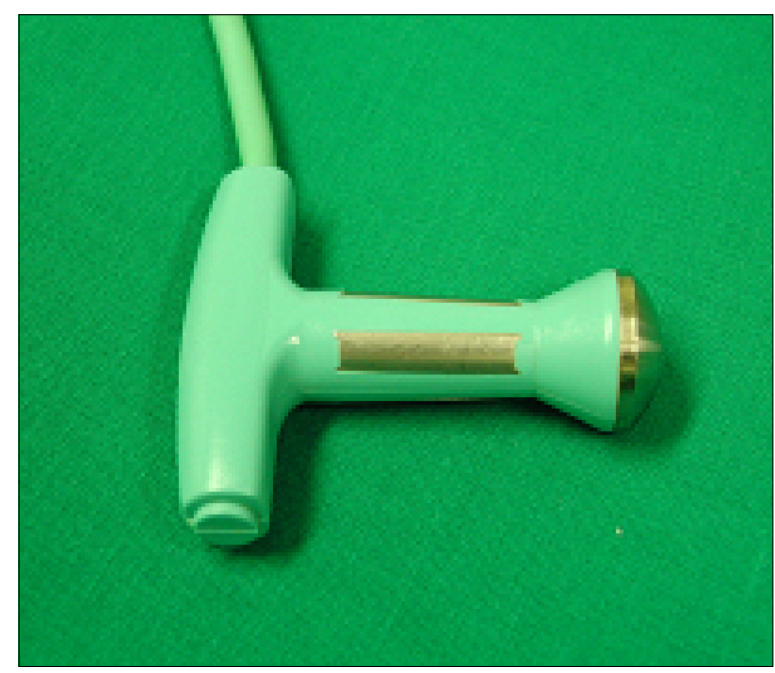

Figure 2. An anorectal probe with pulse generator. 


\section{Results}

\section{Demographic Results}

A total of 159 patients (62 male, 97 female; mean age \pm SD, $53 \pm 17$ years) underwent EST since 2002. EST was successful in 87 patients ( 33 males, 54 females; mean age $\pm \mathrm{SD}, 50 \pm 17$ years) and unsuccessful in 60 patients ( 28 males, 32 females, mean age $\pm \mathrm{SD}, 55 \pm 17$ years), whereas 12 patients ( 5 males, 7 females, mean age $\pm \mathrm{SD}, 55 \pm 17$ years) dropped out. Of the total cohort, 4 patients underwent more than one cycle of EST, with 1 undergoing 7 cycles (Fig. 1).

By intention-to-treat (ITT) analysis, the success rate of EST might be estimated as $54.7 \%$ (87/159), while by per-protocol analysis, the success rate was $59.2 \%$ (87/147).

EST was done using an anorectal probe with pulse generator (Fig. 2).

\section{Indication of Electrical Stimulation Therapy}

The indications for EST were functional constipation refractory to BFT without RH (88 patients, 29 males, 59 females; mean age $\pm \mathrm{SD}, 49 \pm 17$ years) and those with RH (59 patients, 32 males, 27 females; mean age $\pm \mathrm{SD}, 54 \pm 17$ years).

\section{Subjective and Objective Parameters: Overall Results}

\section{Pretreatment results}

Pretreatment subjective parameter (Table 1) and objective (Table 2) parameters, including anorectal manometry, did not differ significantly between the EST-responsive and non-responsive groups.

\section{Changes in Subjective and Objective Para- meters}

In the EST-responsive group, overall satisfaction improved significantly (from $7.3 \pm 3.0$ to $4.3 \pm 2.5, P<0.05$ ), as did other subjective parameters, including feeling of incomplete evacuation, straining, and feeling of obstruction $(P<0.05)$ (Table 3$)$. In addition, objective parameters, such as resting anal sphincter pressure, also decreased significantly $(P<0.05)$ (Table 4). When we compared the EST-responsive and non-responsive groups, the only significant difference in characteristics was maximum volume of sense $(152 \pm 50 \mathrm{mmHg}$ vs. $179 \pm 62 \mathrm{mmHg}$, $P<0.05)$

\section{Factors That Affect the Effectiveness of Elec- trical Stimulation Therapy}

When we analyzed the factors that may influence the effect of EST, including underlying disease, CTT results, subjective pa-

Table 1. Baseline Subjective Parameters in the Groups Responsive and Non-responsive to Electrical Stimulation Therapy

\begin{tabular}{|c|c|c|c|}
\hline & Responsive group $(\mathrm{n}=87)$ & Non-responsive group $(\mathrm{n}=60)$ & $P$-value \\
\hline Age (yr) & $52.3 \pm 16.8$ & $52.7 \pm 16.9$ & NS \\
\hline $\operatorname{Sex}(M / F)$ & $34 / 54$ & $28 / 32$ & NS \\
\hline Satisfaction before $\mathrm{EST}^{\mathrm{a}}$ & $8.3 \pm 2.2$ & $6.3 \pm 3.8$ & NS \\
\hline Satisfaction after EST ${ }^{a}$ & $4.0 \pm 2.0$ & $6.7 \pm 3.2$ & $<0.05$ \\
\hline Medication (n) & 32 & 6 & NS \\
\hline Straining during defecation ${ }^{\mathrm{b}}$ & $8.1 \pm 3.0$ & $6.1 \pm 4.5$ & NS \\
\hline Tenesmus ${ }^{b}$ & $6.6 \pm 3.4$ & $5.7 \pm 4.0$ & NS \\
\hline Obstructive feeling during defecation ${ }^{\mathrm{b}}$ & $7.3 \pm 3.7$ & $7.0 \pm 4.2$ & NS \\
\hline Enema $^{c}$ & $1.2 \pm 1.3$ & $1.1 \pm 1.5$ & NS \\
\hline Desire to defecate $^{\mathrm{d}}$ & $1.9 \pm 1.3$ & $1.9 \pm 1.5$ & NS \\
\hline Frequency of defecation $^{\mathrm{e}}$ & $0.8 \pm 0.7$ & $1.1 \pm 0.8$ & NS \\
\hline Duration of defecation $^{\mathrm{f}}$ & $2.2 \pm 1.4$ & $1.9 \pm 1.4$ & NS \\
\hline
\end{tabular}

${ }^{a}$ Overall satisfaction ( 0 : very unsatisfactory, 10 : very satisfactory), ${ }^{b}$ Straining, obstructive feeling during defecation, Tenesmus $\left(0:\right.$ absent, 10 : very severe), ${ }^{c}$ Enema $(0$ : none, 1 : $<25 \%$ of defecation, $2:>25 \%$ of defecation, $3:$ always), ${ }^{\mathrm{d}}$ Desire of defecation (0: many/day, 1: 1-2/day, 2: 2-3/week, $3: 1 /$ week-10 days, $4:$ none), ${ }^{\mathrm{e}}$ Frequency of defecation (0:3/week, 1: 1-2/week, 2: $<1 /$ week-10 days, $3:<1 / 10$ days), ${ }^{f}$ Duration of defecation $(0:<5$ minutes, $1: 5-10$ minutes, $2: 10-20$ minutes, $3: 20-30$ minutes, $4:>30$ minutes)

EST, electrical stimulation therapy.

Data are presented as mean $\pm \mathrm{SD}$, except sex and medication. 
Table 2. Baseline Objective Parameters in Patients Responsive and Non-responsive to Electrical Stimulation Therapy

\begin{tabular}{lccc}
\hline & Responsive group $(\mathrm{n}=87)$ & Non-responsive group $(\mathrm{n}=60)$ & $P$-value \\
\hline Resting pressure $(\mathrm{mmHg})$ & $143.9 \pm 66.1$ & $127.1 \pm 49.2$ & NS \\
Squeezing pressure $(\mathrm{mmHg})$ & $238.2 \pm 73.9$ & $254.1 \pm 172.6$ & NS \\
Sustained duration $(\mathrm{sec})$ & $5.7 \pm 3.4$ & $10.8 \pm 24.0$ & NS \\
Sphincter length $(\mathrm{cm})$ & $4.1 \pm 0.8$ & $4.1 \pm 0.8$ & NS \\
High pressure zone length $(\mathrm{cm})$ & $2.3 \pm 0.7$ & $2.3 \pm 0.7$ & NS \\
Rectoanal inhibitory reflex & $17.7 \pm 10.5$ & $21.4 \pm 12.2$ & NS \\
Minimum volume of sense $(\mathrm{mL})$ & $13.1 \pm 12.2$ & $16.3 \pm 23.5$ & NS \\
Desire to defecate volume $(\mathrm{mL})$ & $71.9 \pm 44.8$ & $87.7 \pm 50.3$ & NS \\
Urge to defecate volume $(\mathrm{mL})$ & $122.4 \pm 57.8$ & $139.5 \pm 52.2$ & NS \\
Maximum volume $(\mathrm{mL})$ & $152.2 \pm 50.1$ & $179.6 \pm 61.9$ & $<0.05$ \\
Balloon expulsion $(\mathrm{n})$ & 45 & 27 & NS
\end{tabular}

Data are presented as mean $\pm \mathrm{SD}$, except balloon expulsion.

Table 3. Changes in Subjective Parameters in Patients Responsive and Non-responsive to Electrical Stimulation Therapy

\begin{tabular}{|c|c|c|c|c|c|c|}
\hline & \multicolumn{3}{|c|}{ Responsive group $(\mathrm{n}=87)$} & \multicolumn{3}{|c|}{ Non-responsive group $(\mathrm{n}=60)$} \\
\hline & Before EST & After EST & $P$-value & Before EST & After EST & $P$-value \\
\hline Satisfaction $^{\mathrm{a}}$ & $8.3 \pm 2.2$ & $4.0 \pm 2.0$ & $<0.01$ & $6.3 \pm 3.8$ & $6.7 \pm 3.2$ & NS \\
\hline Straining ${ }^{\mathrm{b}}$ & $8.0 \pm 3.0$ & $4.9 \pm 2.9$ & $<0.01$ & $6.1 \pm 4.5$ & $5.5 \pm 4.3$ & NS \\
\hline Tenesmus $^{\mathrm{b}}$ & $6.6 \pm 3.4$ & $3.2 \pm 2.8$ & $<0.01$ & $5.7 \pm 4.0$ & $5.3 \pm 3.7$ & NS \\
\hline Desire of defecation $^{\mathrm{d}}$ & $1.9 \pm 1.3$ & $1.2 \pm 0.8$ & $<0.01$ & $1.9 \pm 1.5$ & $1.4 \pm 1.0$ & NS \\
\hline Frequency of defecation $^{\mathrm{e}}$ & $0.8 \pm 0.7$ & $0.2 \pm 0.5$ & $<0.01$ & $1.1 \pm 0.8$ & $0.5 \pm 0.7$ & $<0.01$ \\
\hline Duration of defecation $^{\mathrm{f}}$ & $2.2 \pm 1.4$ & $1.3 \pm 1.0$ & $<0.01$ & $1.9 \pm 1.4$ & $1.6 \pm 1.1$ & NS \\
\hline Obstructive feeling during defecation $^{\mathrm{b}}$ & $7.3 \pm 3.7$ & $3.3 \pm 3.1$ & $<0.01$ & $7.0 \pm 4.2$ & $6.4 \pm 3.8$ & NS \\
\hline Enema $^{c}$ & $1.1 \pm 1.3$ & $0.2 \pm 0.6$ & $<0.01$ & $1.0 \pm 1.4$ & $0.5 \pm 1.1$ & NS \\
\hline
\end{tabular}

${ }^{\mathrm{a}}$ Overall satisfaction ( 0 : very unsatisfactory, 10 : very satisfactory), ${ }^{\mathrm{b}}$ Straining, obstructive feeling during defecation and tenesmus $\left(0\right.$ : absent, 10 : very severe), ${ }^{\mathrm{c}}$ Enema ( $0:$ none, $1:<25 \%$ of defecation, 2: $>25 \%$ of defecation, 3: always), ${ }^{\mathrm{d}}$ Desire of defecation (0: many/day, 1: 1-2/day, 2: 2-3/week, 3:1/week-10 day, 4: none), ${ }^{\mathrm{e}}$ Frequency of defecation $(0: 3 /$ week, $1: 1-2 /$ week, $2:<1 /$ week-10 days, $3:<1 / 10$ days $),{ }^{\mathrm{f}}$ Duration of defecation $(0:<5$ minutes, $1: 5-10$ minutes, $2: 10-20$ minutes, 3: 20-30 minutes, $4:>30$ minutes).

EST, electrical stimulation therapy.

Data are presented as mean $\pm \mathrm{SD}$.

rameters, and anorectal manometric results, we found that none was significant when comparing the EST-responsive and non-responsive groups (Table 1, 2 and 5). Of the underlying diseases tested, such as diabetes mellitus, hypertension, neurologic disorders, psychiatric disorders, benign prostate hyperplasia, and hypothyroidism, none predicted response to EST (data not shown).

\section{Subgroup Analysis}

\section{Refractory to biofeedback therapy}

Of the 88 patients refractory to BFT, 57 (64.8\%) responded to EST. Of the subjective and objective parameters assessed, none changed significantly between patients responsive and non-responsive to EST except for satisfaction score $(3.8 \pm 2.1$ vs. $6.7 \pm 3.5, P<0.05)$. There were no differences between responsive and non-responsive patients in analysis of CTT $(P>$ 0.05 by Fisher's exact test).

\section{Rectal hyposensitivity}

Of the 59 RH patients, 30 (50.8\%) showed improvement after EST (Table 6). Patients responsive to EST showed significant improvements in desire to defecate volume (DDV; from $115.0 \pm 17.9 \mathrm{~mL}$ to $80.0 \pm 45.6 \mathrm{~mL}, P<0.05)$, urge to defecate volume (UDV; from $178.2 \pm 50.7 \mathrm{~mL}$ to $153.6 \pm 58.0 \mathrm{~mL}$, $P<0.05$ ), and maximum tolerable volume (MTV; from 198.3 \pm 35.1 to $173.3 \pm 37.3$ in MTV, $P<0.05$ ) (Table 7).

When we compared the 30 responders and 29 non-responders to EST, there were no significant differences in the analysis of CTT ( $P>0.05$ by Fisher's exact test) (data not shown). 
Table 4. Changes in Anorectal Manometry Findings in Patients Responsive and Non-responsive to Electrical Stimulation Therapy

\begin{tabular}{|c|c|c|c|c|c|c|}
\hline & \multicolumn{3}{|c|}{ Responsive group $(\mathrm{n}=87)$} & \multicolumn{3}{|c|}{ Non-responsive group $(\mathrm{n}=60)$} \\
\hline & Before EST & After EST & $P$-value & Before EST & After EST & $P$-value \\
\hline Resting anal sphincter pressure $(\mathrm{mmHg})$ & $52.0 \pm 24.7$ & $52.0 \pm 21.6$ & NS & $58.5 \pm 23.7$ & $113.3 \pm 232.8$ & NS \\
\hline Squeezing anal sphincter pressure $(\mathrm{mmHg})$ & $172.3 \pm 59.4$ & $171.0 \pm 65.5$ & NS & $185.9 \pm 117.2$ & $182.0 \pm 101.9$ & NS \\
\hline Sustained duration (sec) & $5.4 \pm 3.4$ & $5.6 \pm 4.0$ & NS & $6.2 \pm 3.7$ & $6.0 \pm 4.1$ & NS \\
\hline Anal sphincter length $(\mathrm{cm})$ & $4.1 \pm 0.8$ & $4.0 \pm 0.7$ & NS & $4.1 \pm 0.9$ & $4.1 \pm 0.8$ & NS \\
\hline High pressure zone $(\mathrm{cm})$ & $2.5 \pm 0.6$ & $2.2 \pm 0.5$ & NS & $2.5 \pm 0.5$ & $2.4 \pm 0.7$ & NS \\
\hline Rectoanal inhibitory reflex & $19.7 \pm 11.4$ & $16.5 \pm 9.5$ & NS & $19.1 \pm 10.0$ & $16.9 \pm 10.1$ & NS \\
\hline Minimal volume of rectal sense $(\mathrm{mL})$ & $14.1 \pm 16.2$ & $10.3 \pm 1.7$ & NS & $15.3 \pm 11.2$ & $11.3 \pm 5.0$ & NS \\
\hline Desire to defecate volume of rectal sense $(\mathrm{mL})$ & $72.9 \pm 39.7$ & $67.1 \pm 44.8$ & NS & $98.0 \pm 41.3$ & $86.0 \pm 39.2$ & NS \\
\hline Urgency volume of rectal sense (mL) & $133.3 \pm 61.7$ & $127.9 \pm 60.7$ & NS & $156.0 \pm 53.7$ & $150.0 \pm 41.9$ & NS \\
\hline Maximal volume of rectal sense $(\mathrm{mL})$ & $161.9 \pm 49.2$ & $151.0 \pm 53.4$ & NS & $195.4 \pm 59.0$ & $178.5 \pm 46.3$ & NS \\
\hline Balloon expulsion (n) & 72 & 32 & NS & 27 & 10 & NS \\
\hline
\end{tabular}

EST, electrical stimulation therapy.

Data are presented as mean $\pm \mathrm{SD}$, except balloon expulsion

Table 5. Differences in Colon Transit Time in Patients Responsive and Non-responsive to Electrical Stimulation Therapy

\begin{tabular}{lccc}
\hline & Responsive group $(\mathrm{n}=87)$ & Non-responsive group $(\mathrm{n}=60)$ & $P$-value \\
\hline Right CTT $(\mathrm{hr})$ & $9.4 \pm 9.7$ & $9.2 \pm 9.3$ & NS \\
Left CTT $(\mathrm{hr})$ & $19.0 \pm 14.4$ & $17.6 \pm 13.9$ & NS \\
Rectosigmoid CTT $(\mathrm{hr})$ & $11.8 \pm 13.3$ & $11.4 \pm 11.7$ & NS \\
Total CTT $(\mathrm{hr})$ & $39.5 \pm 22.8$ & $37.6 \pm 21.9$ & NS \\
\hline
\end{tabular}

CTT, colon transit time.

Data are presented as mean $\pm \mathrm{SD}$.

Table 6. Anorectal Manometric Findings in Patients with Rectal Hyposensitivity Who Were Responsive or Non-responsive to Electrical Stimulation Therapy

\begin{tabular}{lccc}
\hline & Responsive group $(\mathrm{n}=30)$ & Non-responsive group $(\mathrm{n}=29)$ & $P$-value \\
\hline Resting pressure $(\mathrm{mmHg})$ & $45.3 \pm 19.0$ & $52.3 \pm 18.9$ & NS \\
Squeezing pressure $(\mathrm{mmHg})$ & $182.5 \pm 62.4$ & $166.3 \pm 67.9$ & NS \\
Sustained duration $(\mathrm{sec})$ & $4.5 \pm 3.4$ & $5.2 \pm 4.7$ & NS \\
Sphincter length $(\mathrm{cm})$ & $4.3 \pm 0.8$ & $4.2 \pm 0.7$ & NS \\
High pressure zone length $(\mathrm{cm})$ & $2.5 \pm 0.8$ & $2.4 \pm 0.5$ & NS \\
Rectoanal inhibitory reflex & $21.0 \pm 11.6$ & $25.2 \pm 13.5$ & NS \\
Minimum volume of sense $(\mathrm{mL})$ & $18.0 \pm 19.5$ & $16.6 \pm 11.7$ & NS \\
Desire to defecate volume $(\mathrm{mL})$ & $125.0 \pm 34.9$ & $125.5 \pm 40.2$ & NS \\
Urge to defecate volume $(\mathrm{mL})$ & $175.0 \pm 45.8$ & $175.7 \pm 42.0$ & NS \\
Maximum volume $(\mathrm{mL})$ & $194.8 \pm 35.7$ & $217.5 \pm 50.2$ & NS \\
Balloon expulsion $(\mathrm{n})$ & 15 & 13 & NS \\
\hline
\end{tabular}

Data are presented as mean $\pm \mathrm{SD}$, except balloon expulsion.

\section{Adverse Effects of Electrical Stimulation The- rapy}

Only 2 patients showed anal bleeding during the insertion of the anal plug. The nature of bleeding was oozing. It stopped im- mediately after the removal of the plug. Both of these patients had hemorrhoids, which could be the reason for the bleeding. 
Table 7. Changes in Anorectal Manometry Findings in Patients with Rectal Hyposensitivity Who Were Responsive or Non-responsive to Electrical Stimulation Therapy

\begin{tabular}{|c|c|c|c|c|c|c|}
\hline & \multicolumn{3}{|c|}{ Responsive group $(\mathrm{n}=30)$} & \multicolumn{3}{|c|}{ Non-responsive group $(\mathrm{n}=29)$} \\
\hline & Before EST & After EST & $P$-value & Before EST & After EST & $P$-value \\
\hline Resting anal sphincter pressure $(\mathrm{mmHg})$ & $44.9 \pm 15.4$ & $46.3 \pm 14.4$ & NS & $55.4 \pm 22.3$ & $141.3 \pm 279.9$ & NS \\
\hline Squeezing anal sphincter pressure $(\mathrm{mmHg})$ & $169.6 \pm 56.6$ & $182.4 \pm 61.2$ & NS & $180.1 \pm 83.9$ & $182.4 \pm 62.1$ & NS \\
\hline Anal sphincter length $(\mathrm{cm})$ & $4.4 \pm 1.0$ & $4.4 \pm 0.5$ & NS & $4.3 \pm 1.0$ & $4.0 \pm 0.8$ & NS \\
\hline High pressure zone $(\mathrm{cm})$ & $2.7 \pm 0.8$ & $2.2 \pm 0.6$ & NS & $2.5 \pm 0.5$ & $2.4 \pm 0.7$ & NS \\
\hline Rectoanal inhibitory reflex & $24.3 \pm 13.4$ & $19.3 \pm 10.0$ & NS & $21.8 \pm 9.8$ & $20.0 \pm 11.0$ & NS \\
\hline Minimal volume of rectal sense $(\mathrm{mL})$ & $17.9 \pm 23.9$ & $10.0 \pm 0.0$ & NS & $18.2 \pm 12.5$ & $11.8 \pm 6.0$ & NS \\
\hline Desire to defecate volume of rectal sense (mL) & $115.0 \pm 17.9$ & $80.0 \pm 45.6$ & 0.01 & $115.5 \pm 30.8$ & $100.9 \pm 33.9$ & NS \\
\hline Urgency volume of rectal sense (mL) & $178.2 \pm 50.7$ & $153.6 \pm 58.0$ & 0.03 & $178.2 \pm 42.9$ & $164.5 \pm 39.3$ & NS \\
\hline Maximal volume of rectal sense $(\mathrm{mL})$ & $198.3 \pm 35.1$ & $173.3 \pm 37.3$ & 0.03 & $221.3 \pm 45.2$ & $197.5 \pm 44.6$ & NS \\
\hline
\end{tabular}

EST, electrical stimulation therapy.

Data are presented as mean $\pm \mathrm{SD}$.

\section{Discussion}

We have shown here that EST was effective in the treatment of patients who were refractory to conventional BFT, and those with $\mathrm{RH}$, and anal pain with an unknown origin. Prior to EST, patients refractory to conventional treatment, had no choice except for surgery.

Although EST has been used to treat patients with fecal incontinence, the results have been contradictory and dependent on the instrument used. ${ }^{13}$ Conventional machines and electrodes (e.g., Myomed. Enraf-Nonius NV, Delft, Denmark), using constant voltage and current and an already-established protocol, can easily induce muscular fatigue. In contrast, our system (HMT) used a variant mode designed to overcome muscular fatigue and discomfort. In the variant mode, electrostimulation is the summation of energy of variant vectors from the double-channel system. ${ }^{12}$ Energy per pulse is modified by changing the supplied voltage and electric charge. The electric charge is changed according to supplied voltage and pulse width. The supplied voltage is proportional to the duty and intensity of the current. The intensity of the current is related to the skin resistance of the vagina or anus, and duty is proportional to frequency and pulse width. The energy per pulse determines the treatment cycle through phases, units, and sessions. This innovative variant mode is characterized by hundreds of stimulation programs that can be used to meet various requirements. ${ }^{12}$

Fecal incontinence is the inability to control bowel movements normally, causing stools to leak unexpectedly from the rectum. ${ }^{14}$ Common causes of fecal incontinence include muscular or nervous damage due to weakened anal sphincter function associated with aging or childbirth injury. Similar factors are thought to cause RH, suggesting that EST could be useful in treating this condition. ${ }^{15}$ Most RH patients complain of constipation, but many complain of fecal incontinence. ${ }^{15}$ Thus, EST may be effective in the treatment of both $\mathrm{RH}$ and fecal incontinence.

Electrical stimulation can be categorized into four subgroups $^{14}$ : intramuscular electrodes; intraluminal or mucosal electrodes, as used in our center; serosal electrodes for temporary or external stimulation; and the electroacupuncture method. The intraluminal or mucosal electrode method is thought to have several disadvantages, in that contact between the stimulating electrode and mucosa is not guaranteed when suction electrodes or intraluminal electrodes are used. This can be disregarded in the intrarectal or intra-anal form of EST, because the anal plug is large enough to fit into an ordinary person's rectum and anus.

EST using anal plugs has been used to treat urinary incontinence since the 1970s. ${ }^{6}$ Low frequencies $(10-20 \mathrm{~Hz})$ are used to treat urge incontinence and high frequencies $(35-50 \mathrm{~Hz})$ are used to treat stress incontinence. ${ }^{6}$ Little is known, however, about the effectiveness of EST using anal plugs in anorectal functional disorders, especially in patients with fecal incontinence. ${ }^{7,8,12}$

Unfortunately, we could not find any significant differences in our transit time study. We even tried to find a statistical difference by comparing "pure" PFD with a PFD mixed with a slow transit time, but the differences were still not statistically different. We postulated that the reasons might be an insufficient number of enrolled patients to show the statistical difference, or possibly the enrolled patients had constipation that was too complicated to be categorized into a pattern by transit tests alone. 
We found no changes in objective parameters in the overall patient group and in the subgroup of patients refractory to BFT, except for the subgroup of $\mathrm{RH}$ patients. In the latter, objective parameters, including DDV and MTV, were improved after treatment. When we analyzed subjective parameters in the overall patient EST group and subgroups, we found no characteristic that predicted responsiveness to EST.

In conclusion, we found that EST was successful in the treatment of patients with chronic functional constipation refractory to BFT, RH, and anal pain. Factors predictive of responsiveness to EST could not be determined, suggesting the need for additional studies of this modality.

\section{References}

1. Caldwell KP. The electrical control of sphincter incompetence. Lancet 1963;2:174-175.

2. Hopkinson BR, Lightwood R. Electrical treatment of anal incontinence. Lancet 1966;1:297-298.

3. Sanmiguel CP, Casillas S, Senagore A, Mintchev MP, Soffer EE. Neural gastrointestinal electrical stimulation enhances colonic motility in a chronic canine model of delayed colonic transit. Neurogastroenterol Motil 2006;18:647-653.

4. Chang HS, Myung SJ, Yang SK, et al. Effect of electrical stimulation in constipated patients with impaired rectal sensation. Int J Colorectal Dis 2003;18:433-438.

5. Chang HS, Myung SJ, Yang SK, et al. Functional constipation with impaired rectal sensation improved by electrical stimulation therapy: report of a case. Dis Colon Rectum 2004;47:933-936.

6. Fandel T, Tanagho EA. Neuromodulation in voiding dysfunction: a historical overview of neurostimulation and its application. Urol Clin North Am 2005;32:1-10.

7. Healy CF, Brannigan AE, Connolly EM, et al. The effects of lowfrequency endo-anal electrical stimulation on faecal incontinence: a prospective study. Int J Colorectal Dis 2006;21:802-806.

8. Terra MP, Dobben AC, Berghmans B, et al. Electrical stimulation and pelvic floor muscle training with biofeedback in patients with fecal incontinence: a cohort study of 281 patients. Dis Colon Rectum 2006;49:1149-1159.

9. Jung KW, Myung SJ, Byeong J. Combined therapy with electrical stimulation and biofeedback in pelvic floor dyssynergia [abstract]. Neurogastroenterol Motil 2007;19(suppl 3):74.

10. Whitehead WE, Wald A, Diamant NE, Enck P, Pemberton JH, Rao SS. Functional disorders of the anus and rectum. Gut 1999; 45(suppl 2):II55-II59.

11. Prokesch RW, Breitenseher MJ, Kettenbach J, et al. Assessment of chronic constipation: colon transit time versus defecography. Eur J Radiol 1999;32:197-203.

12. Bourcier AP, Park KT. Electrical stimulation. In: Bourcier AP, McGuire EJ, Abrams P, eds. Pelvic Floor Disorders. 1st ed. Philadelphia: Elsevier 2004:281-290.

13. Norton C, Burch J, Kamm MA. Patients' views of a colostomy for fecal incontinence. Dis Colon Rectum 2005;48:1062-1069.

14. Lin Z, Sarosiek I, McCallum RW. Gastrointestinal electrical stimulation for treatment of gastrointestinal disorders: gastroparesis, obesity, fecal incontinence, and constipation. Gastroenterol Clin North Am 2007;36:713-734, x-xi.

15. Gladman MA, Lunniss PJ, Scott SM, Swash M. Rectal hyposensitivity. Am J Gastroenterol 2006;101:1140-1151. 